Technical Note

\title{
Local landmark-based registration for fMRI group studies of nonprimary auditory cortex
}

\author{
Dragana Viceic ${ }^{\mathrm{a}, \mathrm{b}, *}$, Ruth Campos $^{\mathrm{a}, \mathrm{b}}$, Eleonora Fornari ${ }^{\mathrm{c}}$, Lucas Spierer ${ }^{\mathrm{b}}$, Reto Meuli ${ }^{\mathrm{c}}$, \\ Stephanie Clarke ${ }^{\mathrm{b}}$, Jean-Philippe Thiran ${ }^{\mathrm{a}}$ \\ a Signal Processing Laboratory (LTS5), Ecole Polytechnique Fédérale de Lausanne (EPFL), Lausanne, Switzerland \\ b Service de Neuropsychologie et de Neuroréhabilitation, Centre Hospitalier Universitaire Vaudois (CHUV), Lausanne, Switzerland \\ ' Department of Radiology, Centre Hospitalier Universitaire Vaudois (CHUV), Lausanne, Switzerland
}

\section{A R T I C L E I N F O}

\section{Article history:}

Received 21 May 2008

Revised 12 July 2008

Accepted 23 July 2008

Available online 8 August 2008

\section{Keywords:}

Auditory cortex

Averaging

fMRI

Registration

Sound recognition

Sound localization

\begin{abstract}
A B S T R A C T
Interindividual functional and structural brain variability is a major problem in group studies, in which very focal activations are expected. Architectonic studies have shown that the human primary auditory area, which is located with a great constancy on Heschl's gyrus, is surrounded by several nonprimary auditory areas with surface areas of $40-310 \mathrm{~mm}^{2}$. The small size of the latter makes them only partially accessible to fMRI group studies, because of imprecision in realignment when using currently available registration procedures. We describe here a new method for sulcal realignment using a non-rigid local landmark-based registration and show its application to the registration of fMRI acquisitions on the supratemporal plane. After an affine global voxel-based registration, which transforms all brains into the same standard space, we propose a non-rigid local landmark-based registration method based on thin-plate splines for matching the two sulci delimiting Heschl's gyrus of a given brain to the corresponding sulci of a reference brain. We show here that, in comparison with global affine and non-rigid approaches, our method leads in group studies to i) a much more precise alignment of Heschl's gyrus; and ii) a putatively optimal superposition of functionally corresponding areas on and around Heschl's gyrus.
\end{abstract}

(C) 2008 Elsevier Inc. All rights reserved.

\section{Introduction}

Similar to fingerprints, the adult human brain exhibits significant interindividual variability in its global structure, such as in overall size and shape, and also in its local structures, such as in the configuration, length, number and location of gyri and sulci (Amunts et al., 2000, 1999; Geyer et al., 1999; Penhune et al., 1996; Rademacher et al., 1993). The major advances in functional imaging are due to the establishment of statistical methods that allow comparison of individually brains (Mazziotta et al., 2001, 1995; Roland and Zilles, 1994; Van Essen, 2005). The need to locate precisely the site of functional activation within an anatomic framework contributed to the considerable expansion of registration methods. The purpose of interindividual registration is to minimize or remove structural variability to achieve better correspondence of the functionally homologous brain regions across individuals. The most commonly adopted techniques for registering images into the same standard space have two principal characteristics: they are global, considering the entire three-dimensional (3D) brain volume,

\footnotetext{
* Corresponding author. Service de Neuropsychologie et de Neuroréhabilitation, Hôpital Nestlé CHUV, 5, av. Pierre-Decker, CH - 1011 Lausanne, Switzerland. Fax: +41 21 3141319.

E-mail address: Dragana.Viceic@chuv.ch (D. Viceic).
}

and they employ voxel-based registration (VBR), using gray level intensities to evaluate a similarity measure with a template (Collins et al., 1994; Friston et al., 1995; Woods et al., 1992). The drawback of VBR methods is that they are not adapted to match highly variable sulcal patterns.

The human auditory cortex is particularly sensitive to precise registration since it contains, in the vicinity of the primary auditory area, at least six other nonprimary areas that are relatively small, with surface areas between $40 \mathrm{~mm}^{2}$ and $310 \mathrm{~mm}^{2}$ (Rivier and Clarke, 1997; Wallace et al., 2002). A shift of $4 \mathrm{~mm}$ or more in the cortical alignment between corresponding functional areas in individual brains may yield false negative results in group activation studies.

Separate analysis of individual subjects has been recommended to avoid false negative results due to the imprecise realignment of auditory structures (Hashimoto et al., 2000; Schonwiesner et al., 2002; Viceic et al., 2006). However, this approach is time consuming, subjective as to the definition of homologous activation patterns, and lacks the statistical power of group studies.

The need for precise sulcal alignment led to the fast development of the non-rigid local landmark-based registration (LBR) methods (Argall et al., 2006; Desai et al., 2005; Kang et al., 2004). When comparing LBR with global VBR, several authors agree with two facts: i) the local methods are more accurate in precise sulcal superposition than the global methods and ii) non-rigid transformations, such as 
thin-plate splines (TPS) (Kang et al., 2004), fluid deformation (Desai et al., 2005; Van Essen, 2005), and morphing (Desai et al., 2005; Fischl et al., 1999b) provide better interindividual matching than rigid-body or affine transformations. The aforementioned studies have employed two-dimensional (2D) representation of the cortical surface either by cortical inflation to a sphere (Van Essen, 2005) or by flattening to a 2D sheet-surface based atlas (Dale et al., 1999; Fischl et al., 1999a). The cortical inflation and flattening present several drawbacks related to unavoidable distortions of distances and area measurements that they introduce (Fischl et al., 1999a).

The use of local landmark-based registration is most powerful when functional areas have a fixed relationship with distinct anatomical landmarks. This is the case for the primary auditory area, which lies on Heschl's gyrus in most subjects (Rademacher et al., 2001; Zilles et al., 1988).

We present here a new method for a non-rigid local landmarkbased registration (LBR), which allows a more precise interindividual matching of local anatomical structures, such as sulci. In contrast to other methods applying a local registration on the cortical surface, our method is performed in the 3D brain volume. In order to avoid inaccuracy due to the manual selection of landmarks (Kang et al., 2004), we propose a semi-automatic landmark extraction algorithm, where only the identification of the beginning and the end of the sulci of interest is necessary. We did not resort to automatic recognition of sulci as proposed by previous studies (Caunce and Taylor, 2001; Lohmann and von Cramon, 2000; Mangin et al., 1995; Rettmann et al., 2002; Riviere et al., 2002; Royackkers et al., 1999; Vaillant and Davatzikos, 1997). The sulcal pattern of the supratemporal plane is highly variable and the automatic detection fails to identify unequivocally sulci delimiting Heschl's gyrus.

A previous study that performed LBR on the supratemporal region used five anatomical landmarks and observed that in comparison to global VBR, LBR yielded functional maps with improved resolution (Kang et al., 2004). However, to our knowledge no study has examined the effect on functional group studies of sulcal realignment on the supratemporal plane. To assess the performance of our sulcal LBR, we compared it with two other registration methods: a) the single affine global VBR (aVBR) and b) the combination of the affine global VBR and a non-rigid global VBR (VBR), both implemented in Statistical Parametric Mapping (SPM2) software (Wellcome Department of Cognitive Neurology, London, UK; www.fil.ion.ucl.ac.uk/spm; Friston et al., 1994). To illustrate the differences between the three aforementioned methods, we applied them on anatomical and functional datasets of auditory processing on the supratemporal plane involved in sound recognition, "what," and sound localization, "where" pathways (Maeder et al., 2001).

\section{Methods}

A sagittal conventional T1-weighted 3D gradient-echo sequence was acquired for 15 normal volunteers on a 3 Tesla MRI system (Philips Intera, Philips Medical Systems, Best, The Netherlands). Each volume of anatomical MR images consisted of 128 sagittal sections that covered the whole-head volume and that were acquired with following parameters: repetition time $(\mathrm{TR})=9.9 \mathrm{~ms}$, echo time $(\mathrm{TE})=$ $4.6 \mathrm{~ms}$, flip angle $=8^{\circ}$, matrix size $=256 \times 256$, field of view $(F O V)=256$ and slice thickness $=1 \mathrm{~mm}$, yielding isotropic voxels of $1 \mathrm{~mm}^{3}$.

Fifteen normal volunteers participated in this study, aged between 22 and 32 years; eight were female (mean age 24.5 years; $\mathrm{SD}=3.2$ years) and seven were male (mean age 25.6 years; $\mathrm{SD}=1.8$ years). All study participants had normal audition, were right-handed except one, and did not have a history of neurological or psychiatric disorders. A written informed consent was obtained from each participant prior to participation in the study. This study was approved by the Ethical Committee of the Faculty of Biology and Medicine of the University of Lausanne.

\section{Normalization of structural images to the standard space}

The image preprocessing (Fig. 1) was conducted with SPM2 software. To improve the normalization, the anatomical data were first corrected for the intensity inhomogeneity using the bias correction as implemented in SPM2 software. For the normalization to the SPM2 Montreal Neurological Institute (MNI) T1 $2 \times 2 \times 2 \mathrm{~mm}$ template (Friston et al., 1995), registration parameters were estimated and applied on the structural images using a 12-parameter affine transformation as implemented in SPM2 software. At this stage all structural images were transformed into the same standard space. We will refer to this preprocessing as global affine voxel-based registration (aVBR). For the global non-rigid voxel-based registration (VBR), the affine transformation was followed by a non-rigid registration with a transformation modeled by discrete cosine transform basis functions $(7 \times 9 \times 7$ DCT basis $)$.

\section{Local landmark-based registration}

The images that underwent the aVBR were further processed through several steps. First, a smooth pial mesh of each hemisphere representing the boundary of the gray matter-cerebrospinal fluid interface was created with Freesurfer software (http://surfer.nmr. mgh.harvard.edu/) (Fischl et al., 1999b; Talavage et al., 2004).

Second, the sulci of interest were identified and further used as landmarks for the local registration. The anterior limit of the first

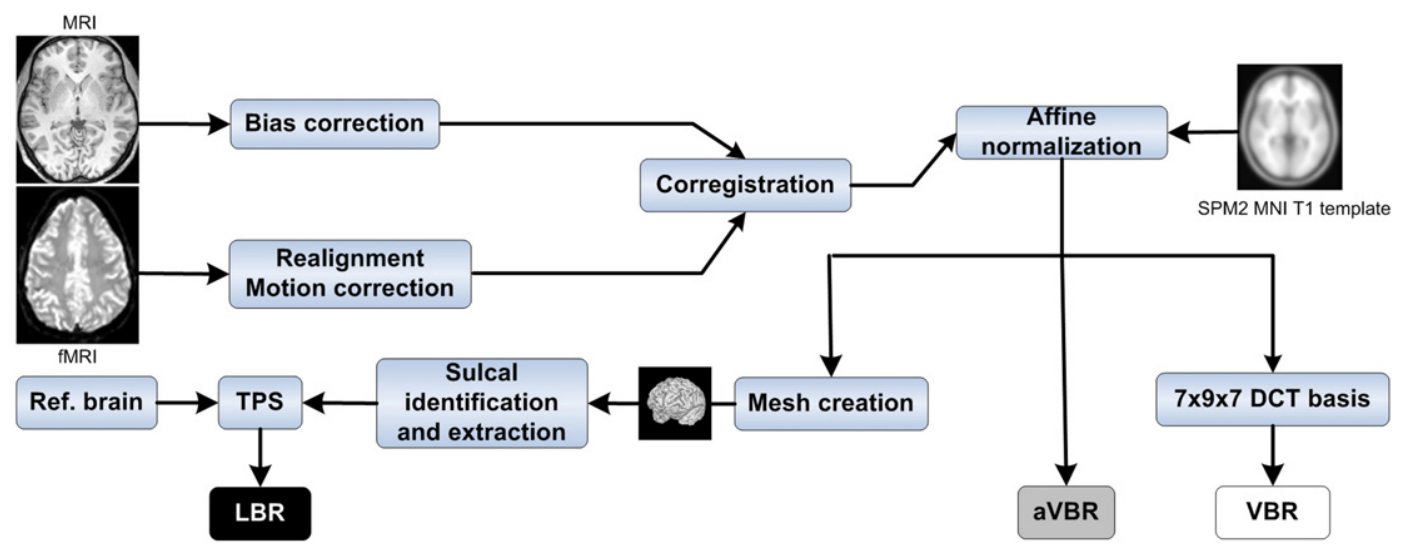

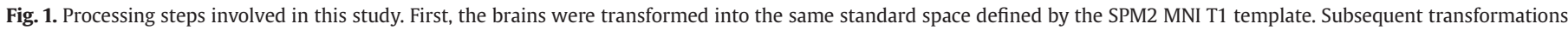

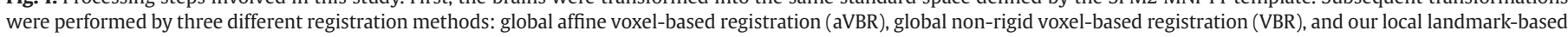
registration (LBR). 
transverse gyrus (TG), i.e., HG, is defined by the first transverse sulcus (FTS). Due to the variable shape of HG, the boundary between the latter and the planum polare, i.e., FTS, was not always precisely and easily delimitated. Therefore, we decided to define the circular sulcus (CirS) as the anterior landmark of HG. In the case of only one HG that was not indented at its crown by an intermediate transverse sulcus (ITS) (Leonard et al., 1998), the posterior limit was determined by the second transverse sulcus, also called Heschl's sulcus (HS). Determining the posterior limit of HG was more delicate in the case of one HG with an ITS, two or three transverse gyri. As an ITS does not completely divide HG in its depth, we decided not to use the ITS as a posterior limit, but the first deep sulcus posterior to it, i.e., HS. In case of two or three transverse gyri that were completely divided by deep sulci, the posterior limit was defined by a HS (Rademacher et al., 2001). Based

\section{A}

Axial plane

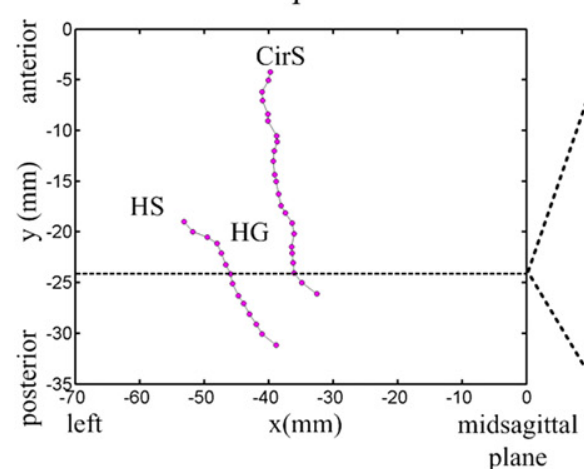

B
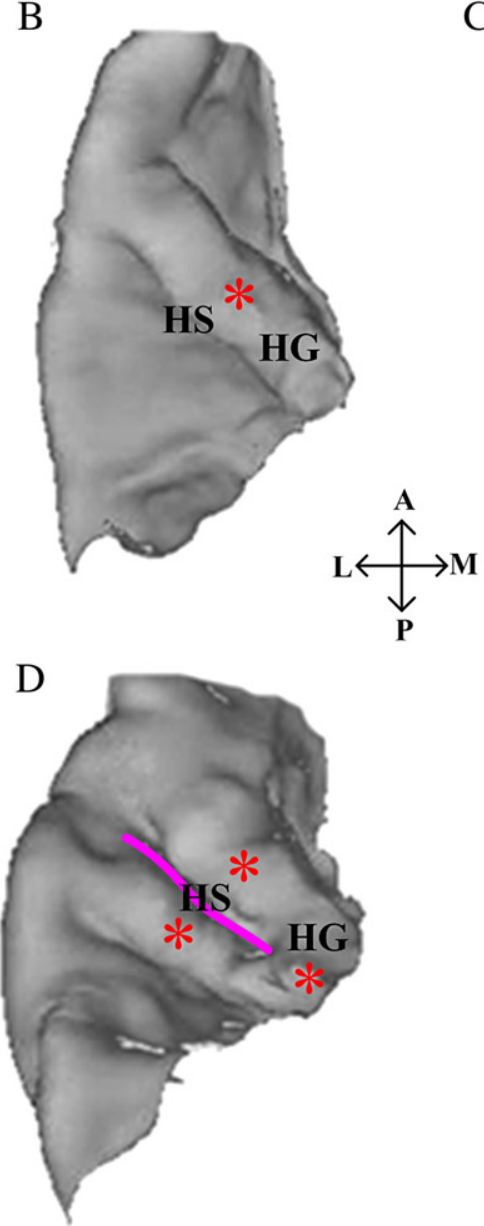

Coronal plane

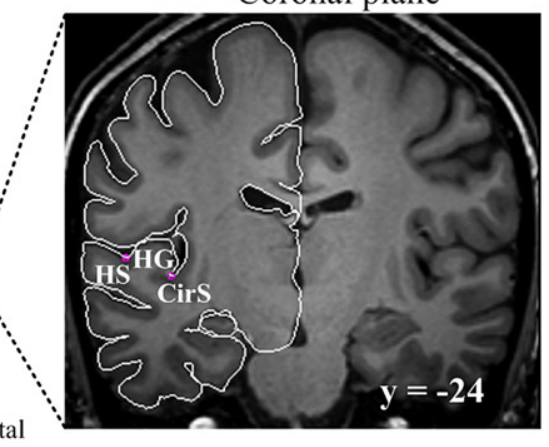

C

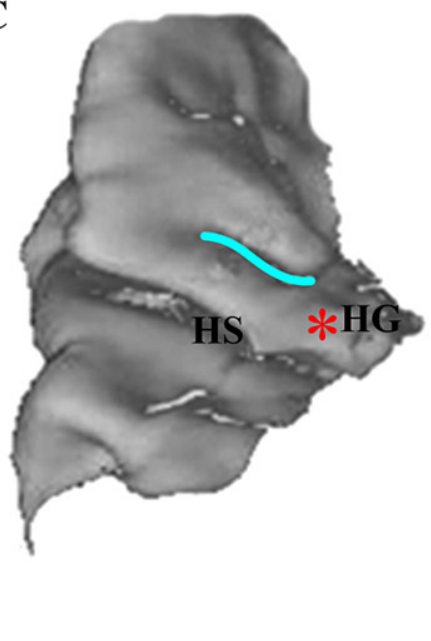

E

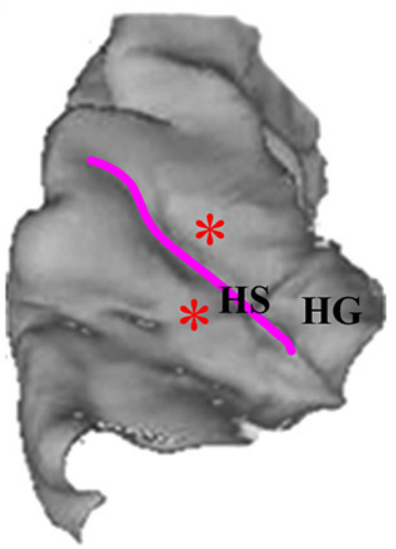

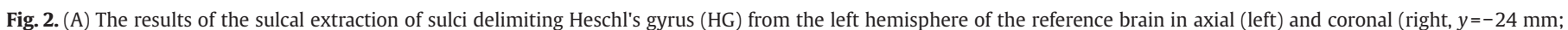

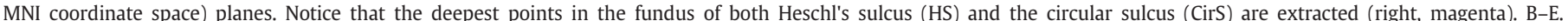

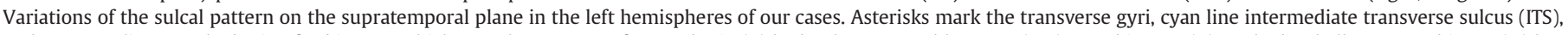

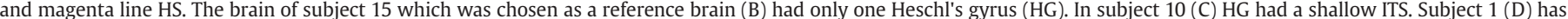

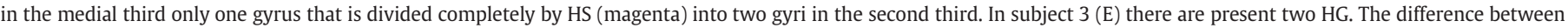

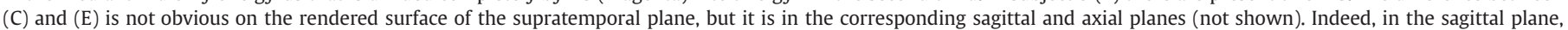

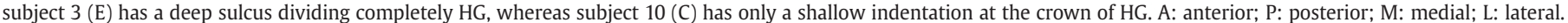


A

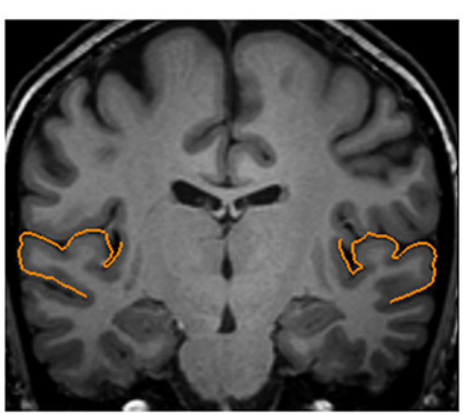

E

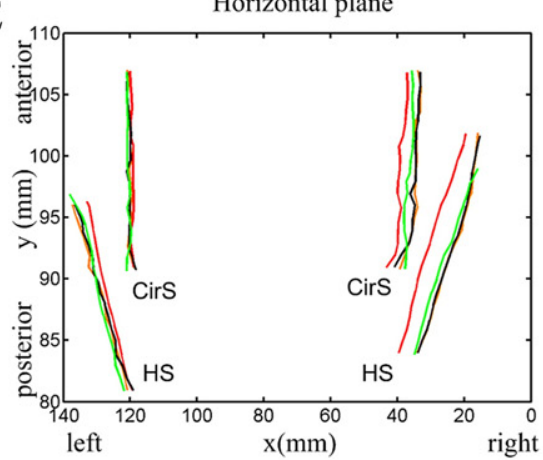

Coronal plane

B

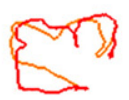

$\mathrm{C}$

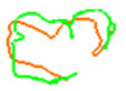

D

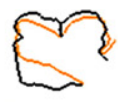

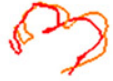
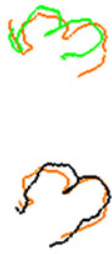

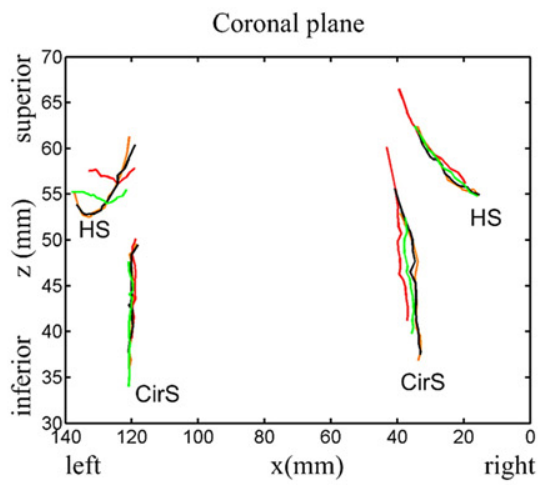

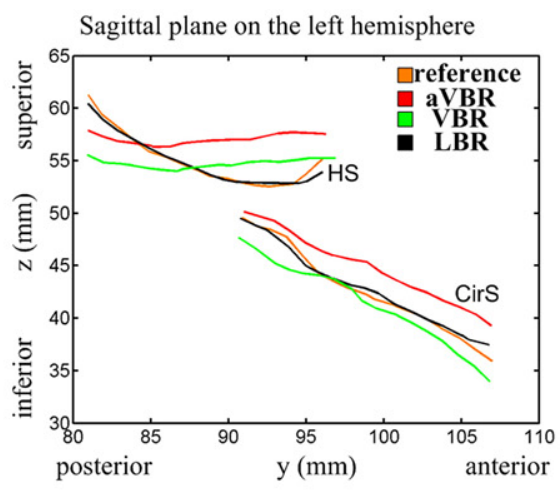

Sagittal plane on the right hemisphere

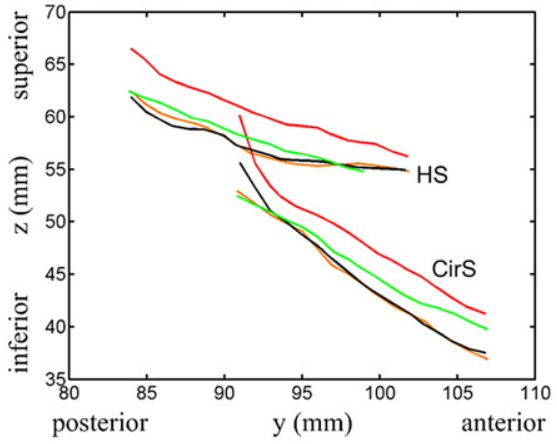

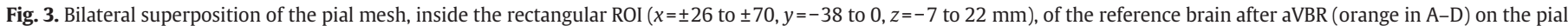

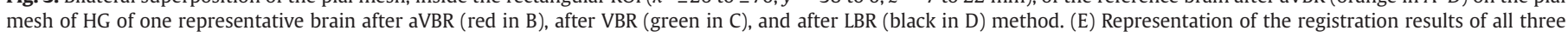
methods in one representative brain in four planes: horizontal, coronal and two sagittal (left and right) planes.

on these considerations, sulcus identification was performed by one of our neuroanatomy experts. All ambiguous cases were specifically reviewed by a second neuroanatomy expert.

Third, after their identification, the sulci were extracted semiautomatically. The initial step was to create a rectangular box enclosing the entire required sulcus. The limits of this rectangular box were defined by the simultaneous display of the coronal plane and the pial mesh (Fig. 2A, right panel). Freesurfer software provides the possibility to assign to each vertex of the mesh its curvature value with the positive and negative curvature values indicating sulcal and gyral regions, respectively (Fischl et al., 1999a). We used the maximum positive curvature value to extract automatically the most inferior landmark of the sulcus of the pial mesh, corresponding to sulcal fundus, in each MRI coronal slice inside de box (Fig. 2A). This approach provided exactly one landmark per sulcus per coronal slice, i.e., about 30 to 35 landmarks for two sulci (CirS and HS) per hemisphere.

Fourth, the reference brain was chosen among the group of subjects using the following criteria: i) presence of a single transverse sulcus in both hemispheres; ii) presence of CirS and HS long enough to allow the registration; iii) sulcal positions that were nearest to the mean of the group. Choosing a reference brain is a debated issue (Hellier et al., 2003; Mangin et al., 2004). Although some studies indicate that the choice of the reference brain has minimal influence on the results (Guimond et al., 2000), we observed (data not shown) that the choice of an outlier can produce major and inappropriate, i.e., unsmooth, transformations for all other brains.

Fifth, the sulci of each individual brain were projected onto the corresponding sulci of the reference brain by projecting, within each slice, the sulcal points of the individual brain onto those of the reference brain. As our landmarks are points and not lines, sulcal projection allowed one-to-one correspondence between two sets of landmarks (one point per each coronal slice and per each sulcus). In cases where an individual brain sulcus was longer than the reference brain sulcus, landmarks situated on its extremity did not have their correspondence and were not matched. By this approach we avoided the problem of the correspondence between the end-points of a sulcus in two different brains, particularly when registering very long and very short sulci.

The first part of local registration is to calculate the displacement between the landmark points of each individual brain and the corresponding landmark points of the reference brain. This displacement is defined as

$\overrightarrow{d_{i}}=\left[\begin{array}{lll}d_{x i} & d_{y i} & d_{z i}\end{array}\right]^{T}$

where $\overrightarrow{d_{i}}=\overrightarrow{q_{i}}-\overrightarrow{p_{i}}, i=1,2, \ldots, N, N$ is number of landmarks, $\overrightarrow{q_{i}}$ and $\overrightarrow{p_{i}}$ are the reference brain and corresponding individual brain landmark $i$, respectively.

Thereafter the displacement field around the landmarks must be interpolated from the displacement of the landmarks. Splines are widely recognized as one of the most successful methods to interpolate the coordinate transformation on intermediate locations, minimizing bending energy (Davis et al., 1997). There are several types of splines: TPS, elastic body splines (EBS), and volume splines (VS). The TPS method is one of the most commonly used methods for landmark registration. It is suitable because it does not need a determination of parameters by the user, as in EBS, and the computation is performed faster in comparison to EBS and VS (Davis et al., 1997). A TPS interpolant, $f(x, y, z)$, minimizes its "bending energy" and is defined for any coordinate of the image by

$f(x, y, z)=a_{0}+a_{x} x+a_{y} y+a_{z} z+\sum_{i=1}^{n} \overrightarrow{w_{i}} G\left(\vec{x}-\vec{p}_{i}\right)$

where $a_{0}+a_{x} x+a_{y} y+a_{z} z$ is the affine portion of the transformation and $G$ is a radial basis function or Green's function that has the form

$G\left(\vec{x}-\vec{p}_{i}\right)=\overrightarrow{r_{i}}$

with $r_{i}^{2}=\left(\mathrm{x}-p_{x i}\right)^{2}+\left(y-p_{y i}\right)^{2}+\left(z-p_{z i}\right)^{2}$, and $\overrightarrow{p_{i}}$ landmarks coordinate. 
Table 1

Number of cases in our population with a single transverse gyrus (1 TG), two (2 TG), or a single one dividing laterally into two ( $1 \mathrm{TG} \rightarrow 2 \mathrm{TG}$ ) in a given hemisphere

\begin{tabular}{lllc}
\hline Number of TG & Left hemisphere $(n)$ & Right hemisphere $(n)$ & Total \\
\hline 1 TG & 11 & 13 & 24 \\
1 TG $\rightarrow 2$ TG & 3 & 0 & 3 \\
2 TG & 1 & 2 & 3 \\
\hline
\end{tabular}

TG: transverse gyrus; $n$ : number of participants.

Each set of features employed for our local registration was composed of 60 to 70 landmarks corresponding to the deepest points of the four sulci on each coronal slice. 175 additional identical landmarks, situated on the regular grid and separated by 30 voxels, were added at the same location in each dataset. They are necessary to ensure that the deformation field remains minimal far from the landmarks. To illustrate the effect of LBR on functional group study and to compare it with the aVBR and VBR methods, we employed functional data on a sound recognition and a sound localization paradigm.

\section{Experimental protocol}

Brain activation associated with sound recognition or sound localization was investigated in each participant with a triple epoch functional magnetic resonance imaging (fMRI) study (sound recognition-sound localization-rest) as described in a previous study (Maeder et al., 2001), with the difference that here we use 3 Tesla MRI system (Philips Intera, Philips Medical Systems, Best, The Netherlands). Sound recognition and sound localization tasks involved both 5-s long presentations of stimuli, composed of sound targets superimposed on a sound background, followed by the acquisition, both were active and required a motor response.

In the recognition task, the stimulus consisted of five different everyday auditory scenes (a market place, street, beach, shop or railway station), within which were imbedded the targets lasting $2 \mathrm{~s}$ and starting $1.5 \mathrm{~s}$ after the stimulation onset (musical instrument or animal cry). The participants were required to press a button on a MRI compatible keyboard with their right index when the target corresponded to an animal cry.

In the localization task, the background consisted of 25 white noise bursts with duration of $50 \mathrm{~ms}$ and interaural time differences varying between 0 and $681 \mu \mathrm{s}$; the latter simulated different azimuthal positions. The targets consisted of two $500 \mathrm{~ms}$ long, filtered white noise tracts (low-pass $1000 \mathrm{~Hz}$ ) lateralized by means of interaural time differences; the first was presented $1.5 \mathrm{~s}$ and the second $3 \mathrm{~s}$ after the onset of the background. Participants were required to press a button with their right index when the two targets were presented in the same location. For more details on the experimental protocol, sound generation, and sound transmission refer to the study of Maeder and colleagues (Maeder et al., 2001).

\section{Functional magnetic resonance imaging}

Functional MRI images were acquired with an echo planar imaging gradient-echo $\mathrm{T} 2 *$-weighted sequence (number of slices $=16$; flip angle $=90^{\circ}$; echo time $=66 \mathrm{~ms}$; slice thickness $=5 \mathrm{~mm}$; interslice gap=1 mm; pixel size $=2.1 \times 2.1 \mathrm{~mm}$; acquisition time= $1.09 \mathrm{~s}$ ). The 16 slices, acquired in a sequential ascending order, covered the wholehead volume in the bicommissural plane. A long repetition time of $15 \mathrm{~s}$ and careful adjustment of the timing of the stimuli presentation allowed acquisition of the fMRI data only during the blood-oxygenlevel dependent (BOLD) effect elicited by the stimuli. The 5-s stimuli were presented in blocks of 5 for each condition (localization and recognition) and were followed by a resting state block; the described sequence was repeated 6 times for a total of 90 acquisitions over $22 \mathrm{~min}$ and $30 \mathrm{~s}$. Additional two acquisitions were acquired at the beginning of each scanning sessions that were discarded during image processing to achieve steady-state magnetization.

In order to correct for head displacement during acquisition, fMRI data were realigned by estimating the six parameters of a rigid-body transformation that minimizes the differences between each successive scan and the first considered scan (Fig. 1). Fourth Degree B-Spline interpolation (Friston et al., 1995) was used for the transformation of the images. The functional images generated after motion correction were coregistered to the structural data. Twelve-parameter affine transformation estimated for structural images was applied on the functional images in order to obtain functional aVBR images. Functional VBR and LBR images were obtained by applying the corresponding transformations estimated for structural images. Finally, all three sets of functional images, after aVBR, VBR and LBR, were resliced to $2 \times 2 \times 2 \mathrm{~mm}$ voxel size and smoothed in order to increase the signal-to-noise ratio using an isotropic Gaussian Kernel of 6 -mm full width at half maximum (FWHM).

B
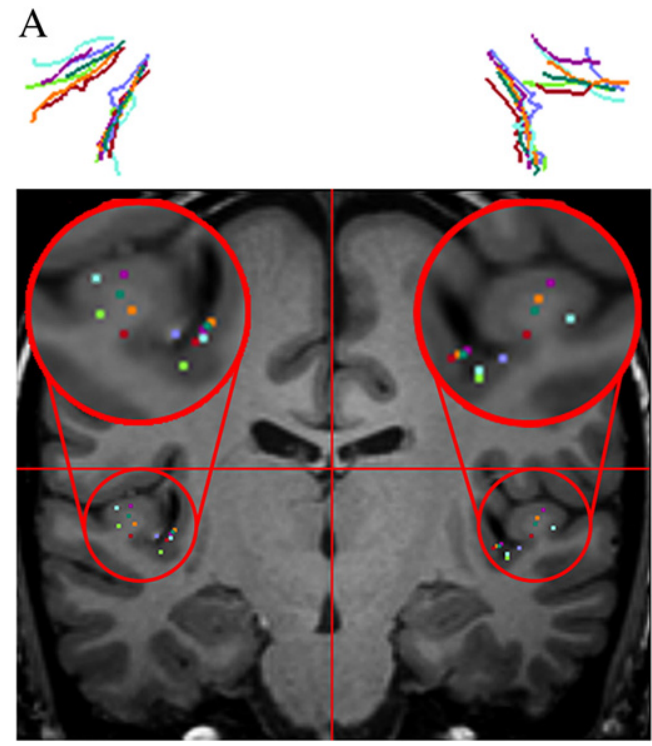
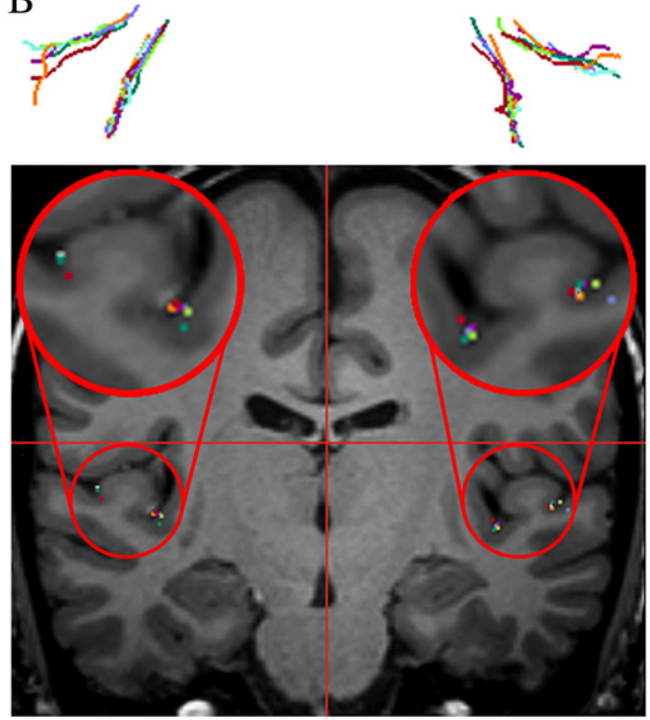

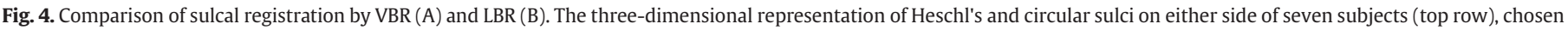

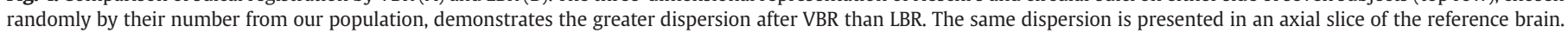

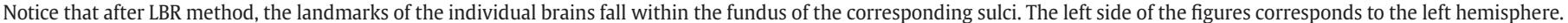




\section{Functional MRI data analyses}

For each participant, we performed first level statistics according to the general linear model as implemented in SPM2 software (Friston et al., 1995). We specified two contrasts of interest: recognition vs. rest and localization vs. rest. Statistical parametric maps (SPM) of the contrasts of interest were computed for each subject as input values
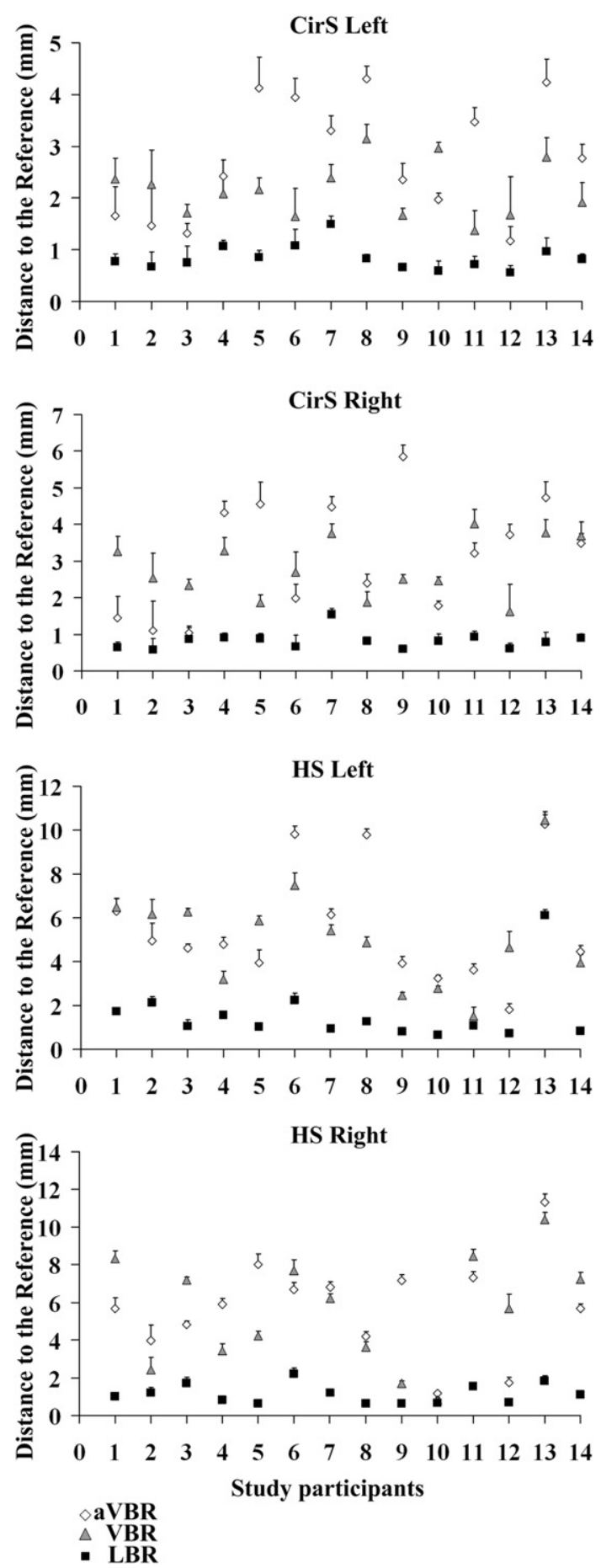

Fig. 5. Comparison of the mean distance to the reference brain for each subject (designated by numbers 1 to 14 ) after the realignment by three different methods for circular sulcus (CirS) in the left (first row) and in the right hemispheres (second row) and Heschl's sulcus (HS) in the left (third row) and in the right (fourth row) hemispheres. aVBR: affine global voxel-based registration; VBR: non-rigid global voxelbased registration; LBR: non-rigid local landmark-based registration. Error bars are standard errors of the mean (SEM). for a second level $t$-statistic based on Gaussian random field theory. The generated SPM maps were thresholded at a $P$ value $<0.05$ corrected at voxel level for multiple comparisons (family-wise error, FWE) across the whole brain (Worsley et al., 1996). Additionally, a cluster extent threshold of 5 voxels $\left(40 \mathrm{~mm}^{3}\right)$ was applied.

\section{Comparison of aVBR, VBR and $L B R$}

The results of all three methods, aVBR, VBR and LBR, were compared in order to evaluate their effect on the anatomical and the functional dataset. First of all, the accuracy of the structural dataset registration of the supratemporal plane was evaluated two-dimensionally by representing the pial mesh of each brain and comparing in to the reference brain, for each of the methods used (aVBR, VBR, or LBR). Graphically, the sulcal realignment of CirS and HS on either side was represented in four views: coronal, axial and two sagittal (left and right).

The mean distance of each individual sulcus of interest in given brain to that of the corresponding sulcus in the reference brain was calculated for each method; it corresponded to the mean value of the Euclidean distance from all landmarks in a given brain to the corresponding ones in the reference brain. The significance of the difference between the three transformations was assessed with paired $t$-tests.

The effect of the three methods on the group results of the fMRI study was evaluated by superimposing thresholded SPM maps of two contrasts $(T>8.44, P<0.05$ corrected for multiple comparisons, cluster extent threshold $k \geq 5$ voxels) on the anatomical image of the reference brain. In order to compare three registrations methods, we calculated the total number of significantly activated voxels in the rectangular ROI defined as $x= \pm 26$ to $\pm 70 \mathrm{~mm}, y=-38$ to $0 \mathrm{~mm}$, and $z=-7$ to $22 \mathrm{~mm}$ in the MNI coordinate space. To quantify which method produces higher $t$-values, we divided the range of the significant $t$-values into eight bins. For each bin, we compared the number of voxels among the three methods and evaluated their statistical difference with paired $t$-tests.

\section{Results}

\section{Population variability}

In $80 \%$ of the hemispheres (24/30) (Table 1$)$, only one TG was present (Fig. 2B); four of these hemispheres had a shallow indentation at the crown of the TG by ITS (Fig. 2C, cyan). Of the remaining $20 \%$ of the hemispheres (6/30), half (3/30) presented with one TG at the medial third, which was divided with a deep sulcus into two transverse gyri when approaching the lateral side of the supratemporal plane (Fig. 2D), while the other half presented with two transverse gyri (Fig. 2E). Our proportions of the four variations described above are consistent with previous structural studies (Morosan et al., 2001; Rademacher et al., 2001).

\section{Structural validation}

For structural validation, the mesh of the supratemporal plane of the reference brain after aVBR (Fig. $3 \mathrm{~A}$, orange line) was compared with the supratemporal plane of the 14 brains that were transformed by aVBR, VBR, or LBR, as shown for a representative brain after aVBR (red line in Fig. 3B), VBR (green line in Fig. 3C), or LBR (black line in Fig. 3D). The LBR yielded better sulcal superposition than aVBR or VBR.

The position of the fundi of HS and of the CirS after registration with any of the three methods has been compared in the four planes of space (horizontal, coronal, sagittal left, and sagittal right; Fig. 3E). After LBR (in black), sulci showed better superposition to the reference sulci (in orange) than after aVBR (in red) or VBR (in green). 


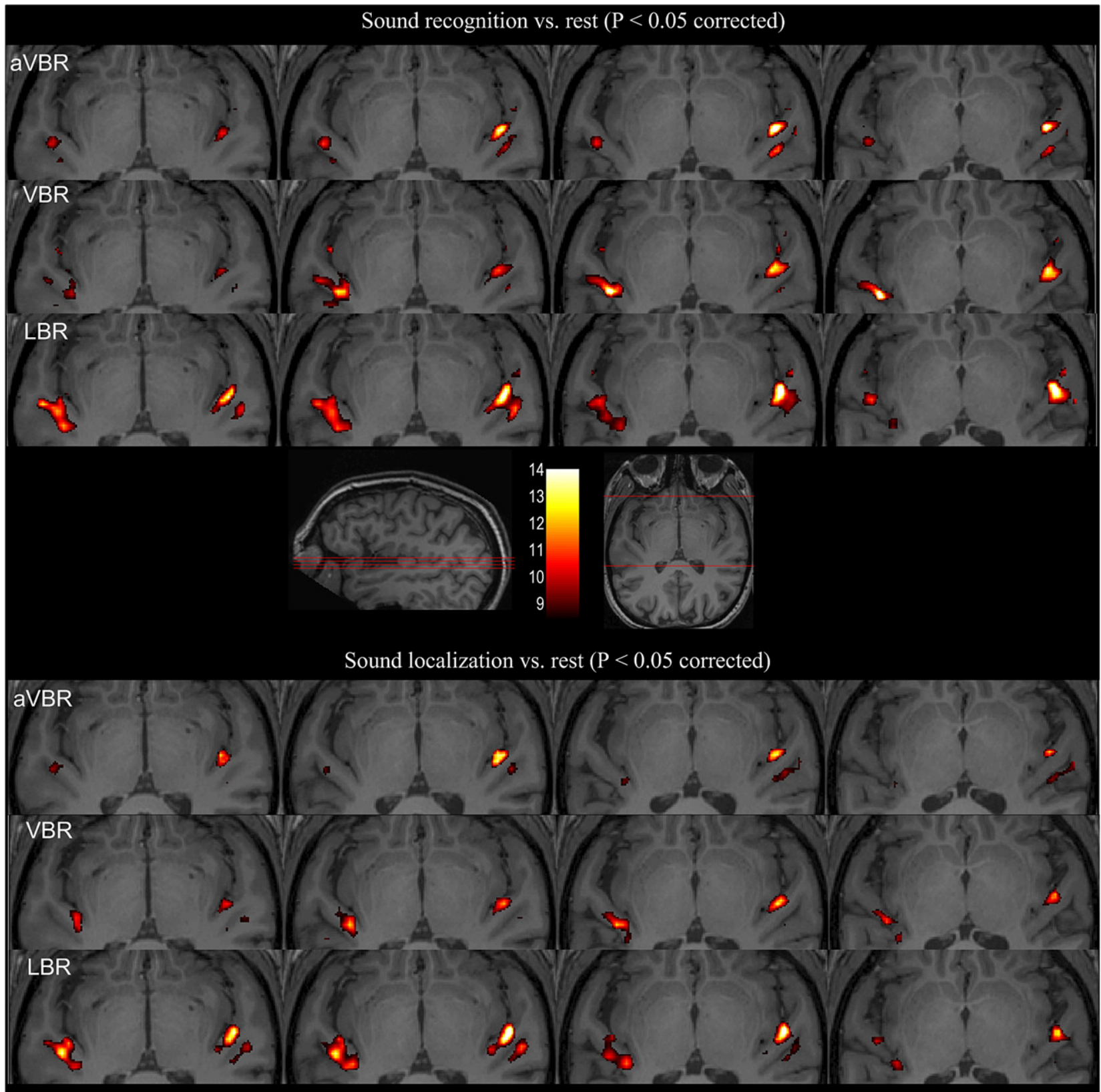

Sound recognition vs. rest $(\mathrm{P}<0.05$ corrected $)$

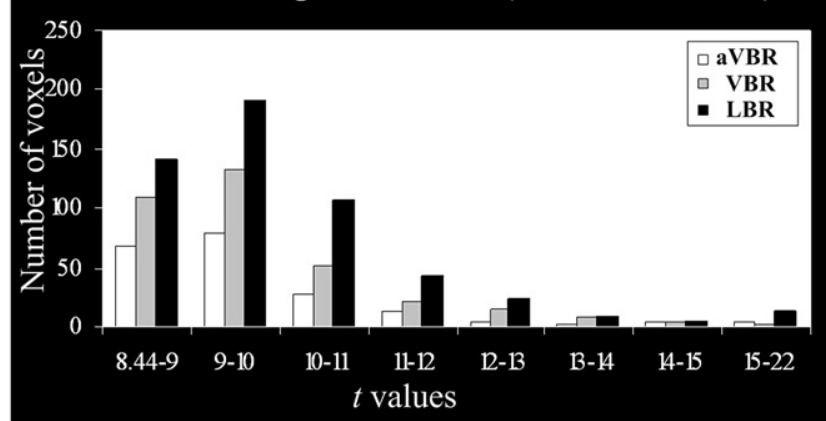

Sound localization vs. rest $(\mathrm{P}<0.05$ corrected $)$

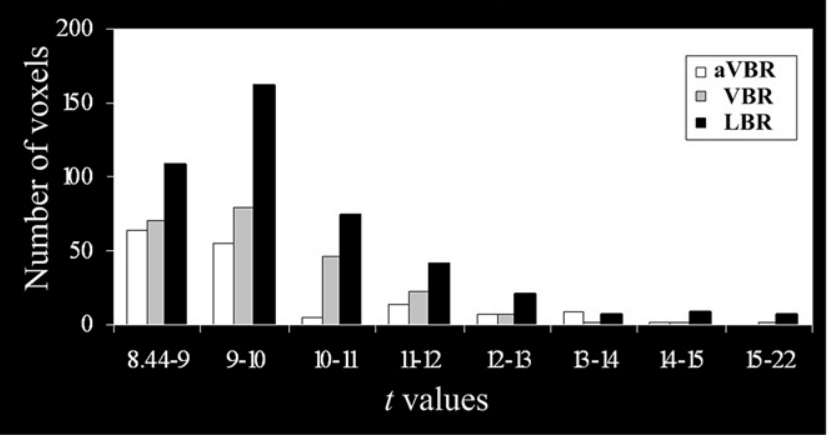

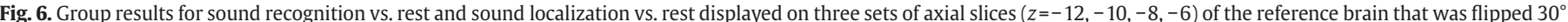

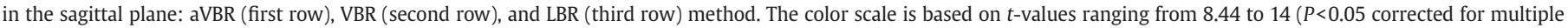

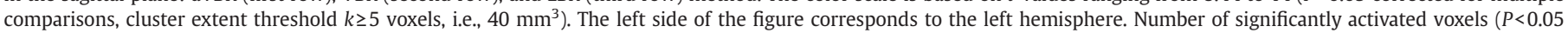
corrected) for eight bins of $t$-values obtained by group mean effect of sound recognition vs. rest (bottom row left) and sound localization vs. rest (bottom row right). 
Table 2

Total number of significantly activated voxels in the rectangular ROI $(x= \pm 26$ to \pm 70 , $y=-38$ to $0, z=-7$ to $22 \mathrm{~mm}$ ) for sound recognition and sound localization after the sulcal realignment with three different methods: aVBR, VBR, and our LBR method

\begin{tabular}{llll}
\hline & aVBR & VBR & LBR \\
\hline Sound recognition vs. rest & 206 & 346 & 532 \\
Sound localization vs. rest & 154 & 232 & 430 \\
\hline
\end{tabular}

The comparison of seven brains, chosen randomly by their number from our population, showed that after LBR (Fig. 4B) the fundi of HS and CirS were more precisely realigned than after VBR (Fig. 4A).

The mean distances of the reference brain landmarks and the corresponding landmarks of each participant were compared after aVBR (white), VBR (gray), or LBR (black) methods (Fig. 5). In all brains the mean distance of both the HS and CirS to those of the reference brains decreased more after LBR than after aVBR or VBR. Paired $t$-tests across different methods for the four sulci showed a significant decrease of the distance after LBR $(P<0.001)$ for all four sulci as compared to aVBR and VBR. A paired $t$-test of mean distance between aVBR and VBR did not demonstrate any statistically significant differences ( $P=0.07$ for CirS left; $P=0.5$ for CirS right; $P=0.43$ for HS left; $P=0.74$ for HS right). The mean distance after the LBR method should be equal to zero according to the definition of TPS declaring that the two sets of landmarks are perfectly superimposed. This difference from zero is due to an error occurring during the pial mesh creation that does not always perfectly fit the cortical surface. Moreover, an intrinsic error of $1 \mathrm{~mm}$ was due to the image resolution.

\section{Functional validation}

Mean group effects of sound recognition and sound localization were compared after the three methods (Fig. 6). Sulcal realignment with LBR provided larger activation clusters with higher $t$-values than did aVBR or VBR. A cluster in the left hemisphere, in both contrasts, obtained by LBR seems to be subdivided in three, or more, smaller clusters with all them situated on HG or just posterior to it. Clusters obtained by aVBR or VBR methods are smaller, and do not have any clear subdivision into smaller clusters. In the right hemisphere LBR method reveals two activation clusters: one cluster situated on HG and one cluster situated posterior to it. This subdivision in two clusters is less obvious after aVBR or VBR methods. Quantitative analyses (Fig. 6, bottom row) revealed that LBR method yielded more statistically significant voxels with high $t$-values in the rectangular ROI $(x= \pm 26$ to $\pm 70 \mathrm{~mm}, y=-38$ to $0 \mathrm{~mm}, z=-7$ to $22 \mathrm{~mm}$ ) when compared to aVBR and VBR for both sound recognition $(\mathrm{LBR}=532, \mathrm{aVBR}=206$, and $\mathrm{VBR}=346)$ and sound localization ( $L B R=430, a V B R=154$, and VBR=232) (Table 2). Paired $t$ tests demonstrated that LBR significantly activated more voxels when compared to aVBR $(P<0.05)$ or VBR $(P<0.05)$ for both sound recognition and sound localization contrasts.

\section{Discussion}

Our method applies a local landmark-based registration using nonrigid transformations to the $3 \mathrm{D}$ brain structure. It has been developed specifically for the supratemporal plane, making use of the well known macro- and microscopical correspondence of HG and the primary auditory area (Rademacher et al., 2001).

Several previous studies have already demonstrated that local landmark-based registration using non-rigid transformations yielded a better superposition than global voxel-based methods either in 2D (Argall et al., 2006; Desai et al., 2005; Kang et al., 2004) or in 3D (Bakker et al., 2008; Kirwan et al., 2007; Miller et al., 2005). A prerequisite for LBR of the supratemporal plane is a good knowledge of the anatomy and histology of this area. Previous anatomical studies demonstrated sulcal variability on the supratemporal plane and notably the presence of one or more transverse gyri (Rademacher et al., 1993). The most frequent case is the presence of one transverse gyrus (70\% of hemispheres; Leonard et al., 1998; Penhune et al., 1996; Rademacher et al., 1993, 2001). In 30 to $50 \%$ of hemispheres, this single transverse gyrus (TG) is indented at its crown by an intermediate transverse sulcus (ITS) (Leonard et al., 1998; Rademacher et al., 1993, 2001). Two transverse gyri are present in $25 \%$ of hemispheres and three transverse gyri in $5 \%$ of hemispheres. In all cases, the anterior landmark of the first TG, i.e., HG, is defined by the FTS. Since the FTS was not always precisely and easily delimitated, we used the CirS as the anterior limit. The definition of the posterior limit was more delicate. Cytoarchitectonic studies of the auditory cortex yielded probability maps showing that the primary auditory cortex is situated on the most anterior and medial part of the first TG extending laterally and posteriorly (Hackett et al., 2001; Rademacher et al., 1993). There was no evident correlation made between the posterior limit of the primary auditory cortex and an ITS or HS. Indeed, in case where HG has an ITS, the primary auditory cortex could be delimited in its posterior part by ITS or by HS (Hackett et al., 2001). As an ITS does not completely divide HG in its depth, we decided not to use the ITS as a posterior limit, but the HS. In case of two or three transverse gyri, cytoarchitectonic studies have shown that the primary auditory cortex was most often limited posteriorly by a HS (Rademacher et al., 2001). Thus, as the posterior limit in all cases, we used the HS, i.e., the first prominent sulcus posterior to the first TG.

For sulcal extraction on the supratemporal plane, we employed an in-house developed algorithm based on semi-automatic sulcal extraction of this region. Thus, we avoided the high computational cost due to the entire brain volume alignment to 3D models of cortical structures (Caunce and Taylor, 2001; Lohmann and von Cramon, 2000; Mangin et al., 1995; Rettmann et al., 2002; Riviere et al., 2002; Royackkers et al., 1999; Vaillant and Davatzikos, 1997) and problems of matching not well identified anatomical features in highly variable regions, such as the auditory cortex. In this study, the sulci were extracted from the pial surface. Another option would be to use the boundary of the gray matter-white matter (GM/WM) interface (Ratnanather et al., 2003). The most appropriate choice for sulcal extraction would be the surface at half distance between pia and GM/ WM border, representing roughly layer IV. With our 3 Tesla acquisitions the cerebrospinal-gray matter interface offered an excellent and unambiguous contrast.

Our method was successful in aligning HG in our population, partially due to the use of a high-field MRI scanner that yields images with a high spatial resolution (Barbier et al., 2002) offering thus more precise spatial information for landmark-based registration.

By aligning precisely HG and hence the primary auditory area, we achieved putatively a better alignment of the primary and nonprimary auditory areas. Assuming that functional organization is correlated to anatomical landmarks, what was established for primary sulci (Hasnain et al., 2006), more precise landmark registration should lead to more accurate statistical maps of the second level statistics, i.e., group studies, and provide thus more information about the functional specialization of auditory nonprimary areas. Our fMRI results on the auditory "what" and "where" pathways support this interpretation, since the group analysis performed after realignment by the LBR method revealed larger activation clusters with possible subdivision and with higher $t$-values than those obtained with the aVBR or VBR method. The cluster sizes were comparable with those of surface areas of the nonprimary auditory areas as determined histologically (40 $\mathrm{mm}^{2}$ to $310 \mathrm{~mm}^{2}$; Rivier and Clarke, 1997; Wallace et al., 2002). The increase in $t$-values is suggestive of functional specialization of these areas as demonstrated previously in single subject analysis (Viceic et al., 2006). The visualization of more than one functional cluster on the supratemporal plane per hemisphere, as it was revealed with our LBR method, is consistent with histological 
studies that intimated the existence of functional subdivisions within the primary auditory cortex (Clarke and Rivier, 1998; Morosan et al., 2001) and of several functional areas outside it (AA, ALA, MA, PA, LA and STA; Rivier and Clarke, 1997; Wallace et al. 2002). In the future, this method may find implication in studies where functional specialization of small areas has to be defined and where structuralfunctional correlation exists.

\section{Acknowledgments}

This study was supported by grants from Max Cloëtta Foundation 3236B0-108530 to DV and by the Swiss National Science Foundation 31000AO-103895 to SC.

\section{References}

Amunts, K., Schleicher, A., Burgel, U., Mohlberg, H., Uylings, H.B., Zilles, K., 1999. Broca's region revisited: cytoarchitecture and intersubject variability. J. Comp. Neurol. 412, 319-341.

Amunts, K., Malikovic, A., Mohlberg, H., Schormann, T., Zilles, K., 2000. Brodmann's areas 17 and 18 brought into stereotaxic space-where and how variable? Neuroimage 11, 66-84.

Argall, B.D., Saad, Z.S., Beauchamp, M.S., 2006. Simplified intersubject averaging on the cortical surface using SUMA. Hum. Brain Mapp. 27, 14-27.

Bakker, A., Kirwan, C.B., Miller, M., Stark, C.E., 2008. Pattern separation in the human hippocampal CA3 and dentate gyrus. Science 319, 1640-1642.

Barbier, E.L., Marrett, S., Danek, A., Vortmeyer, A., van Gelderen, P., Duyn, J., et al., 2002 Imaging cortical anatomy by high-resolution MR at 3.0T: detection of the stripe of Gennari in visual area 17. Magn. Reson. Med. 48, 735-738.

Caunce, A., Taylor, C.J., 2001. Building 3D sulcal models using local geometry. Med. Image Anal. 5, 69-80.

Clarke, S., Rivier, F., 1998. Compartments within human primary auditory cortex: evidence from cytochrome oxidase and acetylcholinesterase staining. Eur. J. Neurosci. 10, 741-745.

Collins, D.L., Neelin, P., Peters, T.M., Evans, A.C., 1994. Automatic 3D intersubject registration of MR volumetric data in standardized Talairach space. J. Comput. Assist. Tomogr. 18, 192-205.

Dale, A.M., Fischl, B., Sereno, M.I., 1999. Cortical surface-based analysis. I. Segmentation and surface reconstruction. Neuroimage 9, 179-194.

Davis, M.H., Khotanzad, A., Flamig, D.P., Harms, S.E., 1997. A physics-based coordinate transformation for 3-D image matching. IEEE Trans. Med. Imaging 16, 317-328.

Desai, R., Liebenthal, E., Possing, E.T., Waldron, E., Binder, J.R., 2005. Volumetric vs. surface-based alignment for localization of auditory cortex activation. Neuroimage 26, 1019-1029.

Fischl, B., Sereno, M.I., Dale, A.M., 1999a. Cortical surface-based analysis. II: Inflation, flattening, and a surface-based coordinate system. Neuroimage 9, 195-207.

Fischl, B., Sereno, M.I., Tootell, R.B., Dale, A.M., 1999b. High-resolution intersubject averaging and a coordinate system for the cortical surface. Hum. Brain Mapp. 8, 272-284.

Friston, K.J., Holmes, A.P., Worsley, K.J., Poline, J.P., Frith, C.D., Frackowiak, R.S.J., 1994. Statistical parametric maps in functional imaging: a general linear approach. Hum. Brain Mapp. 2, 189-210.

Friston, K.J., Ashburner, J., Frith, C.D., Poline, J.B., Heather, J.D., Frackowiak, R.S.J., 1995 Spatial registration and normalization of images. Hum. Brain Mapp. 3, 165-189.

Geyer, S., Schleicher, A., Zilles, K., 1999. Areas 3a, 3b, and 1 of human primary somatosensory cortex. Neuroimage 10, 63-83.

Guimond, A., Meunier, J., Thirion, J., 2000. Average brain models: a convergence study. Comput. Vis. Image Underst. 77, 192-210.

Hackett, T.A., Preuss, T.M., Kaas, J.H., 2001. Architectonic identification of the core region in auditory cortex of macaques, chimpanzees, and humans. J. Comp. Neurol. 441,197-222.

Hashimoto, R., Homae, F., Nakajima, K., Miyashita, Y., Sakai, K.L., 2000. Functional differentiation in the human auditory and language areas revealed by a dichotic listening task. Neuroimage 12,147-158.

Hasnain, M.K., Fox, P.T., Woldorff, M.G., 2006. Hemispheric asymmetry of sulcusfunction correspondence: quantization and developmental implications. Hum. Brain Mapp. 27, 277-287.

Hellier, P., Barillot, C., Corouge, I., Gibaud, B., Le Goualher, G., Collins, D.L., et al., 2003. Retrospective evaluation of intersubject brain registration. Medical Imaging, IEEE Transactions on 22, 1120-1130.

Kang, X., Bertrand, O., Alho, K., Yund, E.W., Herron, T.J., Woods, D.L., 2004. Loca landmark-based mapping of human auditory cortex. Neuroimage 22, 1657-1670.
Kirwan, C.B., Jones, C.K., Miller, M.I., Stark, C.E., 2007. High-resolution fMRI investigation of the medial temporal lobe. Hum. Brain Mapp. 28, 959-966.

Leonard, C.M., Puranik, C., Kuldau, J.M., Lombardino, L.J., 1998. Normal variation in the frequency and location of human auditory cortex landmarks. Heschl's gyrus: where is it? Cereb. Cortex 8, 397-406.

Lohmann, G., von Cramon, D.Y., 2000. Automatic labelling of the human cortical surface using sulcal basins. Med. Image Anal. 4, 179-188.

Maeder, P.P., Meuli, R.A., Adriani, M., Bellmann, A., Fornari, E., Thiran, J.P., et al., 2001. Distinct pathways involved in sound recognition and localization: a human fMRI study. Neuroimage 14, 802-816.

Mangin, JF, Frouin, V, Bloch, I, Régis, J, López-Krahe, J, 1995. From 3D magnetic resonance images to structural representations of the cortex topography using topology preserving deformations. J. Math. Imaging Vis. Volume 5, 297-318.

Mangin, J.F., Riviere, D., Cachia, A., Duchesnay, E., Cointepas, Y., Papadopoulos-Orfanos, D., et al., 2004. A framework to study the cortical folding patterns. Neuroimage 23 (Suppl 1), S129-138.

Mazziotta, J.C., Toga, A.W., Evans, A., Fox, P., Lancaster, J., 1995. A probabilistic atlas of the human brain: theory and rationale for its development. The International Consortium for Brain Mapping (ICBM). Neuroimage 2, 89-101.

Mazziotta, J., Toga, A., Evans, A., Fox, P., Lancaster, J., Zilles, K., et al., 2001. A probabilistic atlas and reference system for the human brain: International Consortium for Brain Mapping (ICBM). Philos. Trans. R Soc. Lond. B Biol. Sci. 356, 1293-1322.

Miller, M.I., Beg, M.F., Ceritoglu, C., Stark, C., 2005. Increasing the power of functional maps of the medial temporal lobe by using large deformation diffeomorphic metric mapping. Proc. Natl. Acad. Sci. U. S. A. 102, 9685-9690.

Morosan, P., Rademacher, J., Schleicher, A., Amunts, K., Schormann, T., Zilles, K., 2001. Human primary auditory cortex: cytoarchitectonic subdivisions and mapping into a spatial reference system. Neuroimage 13, 684-701.

Penhune, V.B. Zatorre, R.J., MacDonald, J.D., Evans, A.C., 1996. Interhemispheric anatomical differences in human primary auditory cortex: probabilistic mapping and volume measurement from magnetic resonance scans. Cereb. Cortex 6, 661-672.

Rademacher, J., Caviness Jr., V.S., Steinmetz, H., Galaburda, A.M., 1993. Topographical variation of the human primary cortices: implications for neuroimaging, brain mapping, and neurobiology. Cereb. Cortex 3, 313-329.

Rademacher, J., Morosan, P., Schormann, T., Schleicher, A., Werner, C., Freund, H.J., et al., 2001. Probabilistic mapping and volume measurement of human primary auditory cortex. Neuroimage 13, 669-683.

Ratnanather, J.T., Barta, P.E., Honeycutt, N.A., Lee, N., Morris, H.M., Dziorny, A.C., et al., 2003. Dynamic programming generation of boundaries of local coordinatized submanifolds in the neocortex: application to the planum temporale. Neuroimage 20, 359-377.

Rettmann, M.E., Han, X., Xu, C., Prince, J.L., 2002. Automated sulcal segmentation using watersheds on the cortical surface. Neuroimage 15, 329-344.

Rivier, F., Clarke, S., 1997. Cytochrome oxidase, acetylcholinesterase, and NADPHdiaphorase staining in human supratemporal and insular cortex: evidence for multiple auditory areas. Neuroimage 6, 288-304.

Riviere, D., Mangin, J.F., Papadopoulos-Orfanos, D., Martinez, J.M., Frouin, V., Regis, J., 2002. Automatic recognition of cortical sulci of the human brain using a congregation of neural networks. Med. Image Anal. 6, 77-92.

Roland, P.E., Zilles, K., 1994. Brain atlases-a new research tool. Trends Neurosci. 17, 458-467.

Royackkers, N., Desvignes, M., Fawal, H., Revenu, M., 1999. Detection and statistical analysis of human cortical sulci. Neuroimage 10, 625-641.

Schonwiesner, M., von Cramon, D.Y., Rubsamen, R., 2002. Is it tonotopy after all? Neuroimage 17, 1144-1161.

Talavage, T.M., Sereno, M.I., Melcher, J.R., Ledden, P.J., Rosen, B.R., Dale, A.M., 2004. Tonotopic organization in human auditory cortex revealed by progressions of frequency sensitivity. J. Neurophysiol. 91, 1282-1296.

Vaillant, M., Davatzikos, C., 1997. Finding parametric representations of the cortical sulci using an active contour model. Med. Image Anal. 1, 295-315.

Van Essen, D.C., 2005. A population-average, landmark- and surface-based (PALS) atlas of human cerebral cortex. Neuroimage 28, 635-662.

Viceic, D., Fornari, E., Thiran, J.P., Maeder, P.P., Meuli, R., Adriani, M., et al., 2006. Human auditory belt areas specialized in sound recognition: a functional magnetic resonance imaging study. Neuroreport 17, 1659-1662.

Wallace, M.N., Johnston, P.W., Palmer, A.R., 2002. Histochemical identification of cortical areas in the auditory region of the human brain. Exp. Brain Res. 143, 499-508.

Woods, R.P., Cherry, S.R., Mazziotta, J.C., 1992. Rapid automated algorithm for aligning and reslicing PET images. J. Comput. Assist. Tomogr. 16, 620-633.

Worsley, K.J., Marrett, S., Neelin, P., Vandal, A.C., Friston, K.J., Evans, A.C., 1996. A unified statistical approach for determining significant voxels in images of cerebral activation. Hum. Brain Mapp. 4, 58-73.

Zilles, K., Armstrong, E., Schleicher, A., Kretschmann, H.J., 1988. The human pattern of gyrification in the cerebral cortex. Anat. Embryol. (Berl). 179, 173-179. 